\title{
Genome
}

\section{An endogenous retrovirus presumed to have been endogenized or relocated recently in a marsupial, the red- necked wallaby}

\begin{tabular}{|c|c|}
\hline Journal: & Genome \\
\hline Manuscript ID & gen-2021-0047.R3 \\
\hline Manuscript Type: & Article \\
\hline $\begin{array}{r}\text { Date Submitted by the } \\
\text { Author: }\end{array}$ & 19-Dec-2021 \\
\hline Complete List of Authors: & $\begin{array}{l}\text { Hayashi, Sakura; Kyoto University, Primate Research Institute } \\
\text { Shimizu, Konami; Noichi Zoological Park of Kochi Prefecture } \\
\text { Honda, Yusuke; Noichi Zoological Park of Kochi Prefecture } \\
\text { Katsura, Yukako; Kyoto University, Primate Research Institute } \\
\text { Koga, Akihiko; Kyoto University, Primate Research Institute }\end{array}$ \\
\hline Keyword: & $\begin{array}{l}\text { albinism, marsupial, endogenous retrovirus, retrotransposon, long } \\
\text { terminal repeat }\end{array}$ \\
\hline $\begin{array}{r}\text { Is the invited manuscript for } \\
\text { consideration in a Special } \\
\text { Issue? : }\end{array}$ & Not applicable (regular submission) \\
\hline
\end{tabular}

\section{SCHOLARONE" \\ Manuscripts}


1 An endogenous retrovirus presumed to have been endogenized or relocated

2 recently in a marsupial, the red-necked wallaby

3

4 Sakura Hayashi ${ }^{1}$, Konami Shimizu ${ }^{2}$, Yusuke Honda ${ }^{2}$, Yukako Katsura ${ }^{1}$, and 5 Akihiko Koga ${ }^{1}$

6

$7 \quad{ }^{1}$ Primate Research Institute, Kyoto University, Inuyama City, Japan

$8 \quad{ }^{2}$ Noichi Zoological Park of Kochi Prefecture, Konan City, Japan 9

10 Keywords: albinism, marsupial, endogenous retrovirus, retrotransposon, long 11 terminal repeat

13 Corresponding author: Akihiko Koga (email: koga.akihiko.5n@kyoto-u.ac.jp) 


\section{Abstract}

15 An albino infant wallaby was born to a mother with the wild-type body color.

16 PCR and sequencing analyses of TYR (encoding tyrosinase, which is essential

17 for melanin biosynthesis) of this albino wallaby revealed a 7.1-kb-long DNA

18 fragment inserted in the first exon. Because the fragment carried long terminal

19 repeats, we assumed it to be a copy of an endogenous retrovirus, which we

20 named walb. We cloned other walb copies residing in the genomes of this

21 species and another wallaby species. The copies exhibited length variation, and

22 the longest copy $(>8.0 \mathrm{~kb}$ ) contained open reading frames whose deduced amino

23 acid sequences were well aligned with those of gag, pol, and env of retroviruses.

24 It is not known through which of the following likely processes the walb copy

25 was inserted into TYR: endogenization (infection of a germline cell by an

26 exogenous virus), reinfection (infection by a virus produced from a previously endogenized provirus), or retrotransposition (intracellular relocation of a provirus). In any case, the insertion into $T Y R$ is considered to have been a recent event on an evolutionary timescale because albino mutant alleles generally do not persist for long because of their deleterious effects in wild circumstances. 


\section{Introduction}

The lifecycle of retroviruses includes reverse transcription of their RNA genome and incorporation of DNA products into the chromosome of their host cells. If the incorporation occurs in a germline cell of an organism, the provirus is inherited by subsequent generations. This initial integration step is termed endogenization, and the provirus harbored since then, as part of the chromosome, is called an endogenous retrovirus (Boeke and Stoye 1997). An endogenous retrovirus may increase the per-cell copy number through two processes (Bannert and Kurth 2006). One is reinfection, that is, a virus particle produced from the provirus infects the same host individual and is endogenized. This may occur if the endogenous retrovirus copy retains all genetic information required for proliferation; however, even if the copy is in a defective form, its reinfection can occur through the provision of necessary factors by an exogenous virus. The other process is retrotransposition, that is, the provirus is transcribed and reverse transcribed, and the DNA produced is integrated into the chromosome of the same cell. The latter is basically the same as the amplification mechanism of long terminal repeat (LTR) retrotransposons. Based on this common mechanism and from similarities in structure, it is widely considered that endogenous retroviruses evolved from LTR retrotransposons and/or vice versa. Recent bioinformatic analyses across kingdoms provide support to the view that retroviruses were derived from LTR retrotransposons via acquisition of genes necessary for leaving the host cell and entering another (Eickbush and Jamburuthugoda 2008; Llorens et al. 2009).

Vertebrate genomes usually contain many families of endogenous retroviruses, where a family is defined as copies that are phylogenetically closely related, and each family consists of a large number of copies. For example, the total copy number of endogenous retroviruses in the human genome is ca. 80,000 (Lander et al. 2001; Paces et al. 2004). However, the vast majority of these copies are considered to have arrived at their current chromosomal positions in the remote past. The age of each provirus can be estimated from the difference in sequences between its LTRs; the LTR sequences are identical to each other when a provirus is newly formed; however, as time passes, differences occur and become larger owing to accumulation of mutations. Surveys of human endogenous retroviruses for the age distribution showed that the vast majority are as old as millions of years or more (Costas 2001; Bannert and Kurth 2006; Vargiu et al. 2016). 
In our recent study, we clarified the genetic cause of an oculocutaneous albinism (red eyes and white coat) that occurred in an animal of the red-necked wallaby (Macropus rufogriseus). We found an extra 7.1-kb-long DNA fragment that was inserted in an exon of a protein-coding gene. The gene was TYR that encodes tyrosinase. Tyrosinase serves as an enzyme that catalyzes the first two reactions of melanin biosynthesis (Körner and Pawelek 1982). The extra fragment was subsequently revealed to be an endogenous retrovirus copy by analyzing this and additionally cloned copies, one of which had the structure of a canonical retrovirus. We propose that, on an evolutionary timescale, the particular provirus found in $T Y R$ has recently been inserted. This proposal is based on two observations: its presence in the albinism-causing mutant gene against which there is strong natural selection, and identical sequences exhibited in its LTRs.

\section{Materials and methods}

\section{Ethics}

This study did not include any animal experiments; the materials used were a surplus of blood samples collected for another purpose and feces naturally defecated. This study involved a recombinant DNA experiment and it was approved in advance by the Recombinant DNA Experiment Safety Committee of Kyoto University (approval number 200421).

\section{Animals and DNA preparation}

In November 2015, an albino female wallaby (named Lily) was born to a mother of wild-type body color (named Ran) in Noichi Zoological Park (Konan City, Japan). Ran, born in February 2014, was legally imported in April 2015 from a kangaroo farm in the Netherlands and was subsequently found to have already been pregnant at the time of arrival. In November 2020, we extracted genomic DNA from a surplus of Lily's blood samples that were collected during emergency surgery. For comparison, in December 2020, we prepared genomic DNA from the feces of a wild-type animal that was born and raised in the same zoo. Hereafter, in this report, the wild-type and albino animals (Fig. 1), as well as their DNA samples, are denoted as WT and AL, respectively.

In July 2021, we extracted, genomic DNA from the feces of a tammar 
103 wallaby (Macropus eugenii). This animal (named Noma) was an adult female 104 kept at Kuragaike Park Zoo (Toyota City, Japan) and exhibited the standard 105 body color of this species.

106

\section{PCR, cloning and sequencing}

108 In most vertebrates hitherto examined, the $T Y R$ gene consists of five exons 109 (denoted as Ex1 to Ex5 hereafter). We conducted BLAST searches of the 110 genome databases of tammar wallaby (Meug_1.0 assembly) and koala

111 (phaCin_unsw_v4.1 assembly), using the human TYR exon sequences (Ensembl 112 ENST00000263321.6) as queries. We drew sequences that corresponded to the 113 exons along with their flanking regions and selected 30-nucleotide blocks that 114 encompassed the entire exon region. We then prepared PCR primers that 115 represented these blocks (see Table S1 for sequences and origins). PCR 116 amplification was performed under the same conditions as those previously 117 described (Koga et al. 2020; Mae et al. 2020). PCR products were cloned into 118 the pUC118 vector and sequenced by the Sanger method. Sequencing primers 119 were prepared step by step based on the sequence data obtained.

\section{Results}

\section{PCR amplification of TYR exons} All five TYR exon regions were amplified (Fig. 2). In the assay for Ex1, while WT yielded a fragment of the expected length $(1.5 \mathrm{~kb})$, two fragments of larger sizes $(8.6 \mathrm{~kb}$ and $1.9 \mathrm{~kb})$ were observed in the lane for AL. There was no detectable size difference between WT and AL in the products for Ex2 to Ex5, and all these fragments exhibited the expected lengths.

\section{Structure of Ex1 PCR products}

132 We cloned the single product from WT (denoted as WT1.5) and the two

133 fragments from AL (AL8.6 and AL1.9) separately into the pUC118 vector, and 134 sequenced their entire insert portions (Fig. 3, Fig. S1).

Human TYR Ex1 contains an 819-bp segment that encodes the first 273 amino acids of the tyrosinase protein. The WT1.5 fragment contained an 819-bp sequence that was aligned with no gaps to the human coding sequence. The reduced amino acid sequence obtained from this 819-bp portion did not contain 
a stop codon and exhibited an identity as high as $82 \%(224 / 273)$. Based on these results, we regarded WT1.5 as a fragment that included Ex1 of the wallaby TYR gene.

The AL8.6 fragment carried a 510-bp sequence that corresponded to the first $510 \mathrm{bp}$ of Ex1, an extra segment of $7137 \mathrm{bp}$, and a 315-bp sequence that corresponded to the last $315 \mathrm{bp}$ of Ex1. The sum of the lengths of the two separate Ex1 regions $(510+315=825 \mathrm{bp})$ was $6 \mathrm{bp}$ longer than the length of Ex1 contained in WT1.5 (819 bp). This was because of the presence of the last six nucleotides of the first Ex 1 part (GACATA) at the head of the second Ex1 part, which is reminiscent of a target site duplication created upon integration. The extra 7137-bp fragment (registered at GenBank with accession number LC631488) contained five open reading frames (ORFs) for 709-, 160-, 218-, 783-, and 315-amino-acid peptides. It was also found that the first and the last 389-bp sequences of the extra 7137-bp fragment were identical to each other, which is reminiscent of an endogenous retrovirus.

The AL1.9 fragment contained the first 510-bp portion of Ex1, a 389-bp sequence identical to the 389-bp terminal regions of the insertion in AL8.6, and the last 315-bp portion of Ex1. A 6-bp duplication of GACATA was also found.

\section{Properties of insertion fragments}

A BLAST search using the 7137-bp inserted fragment as a query sequence returned a high-score hit to TvERV (Baillie and Wilkins 2020) which is a type-

\section{$\mathrm{D}$ endogenous retrovirus of the common brushtail possum Trichosurus} vulpecula, an Australian marsupial. The five ORFs found in the 7137-bp fragment shared a high similarity in reduced amino acid sequence with the genes contained in TVERV. Detailed results are described later as part of the results of the structural analysis of this and additional copies. The possession of ORFs featuring an endogenous retrovirus, along with the repeat structures at and outside the ends, indicates that the 7137-bp fragment is an endogenous retrovirus. It was possible that this particular insertion sequence might be one of multiple copies that descended from a common ancestor. Assuming such a situation, we named the putative multicopy family walb and the particular 7137bp copy found in the red-necked wallaby walb_red01.

A solitary LTR is known to be frequently generated in the host genome by homologous recombination between two LTRs (Vitte and Panaud 2003). The AL1.9 fragment contained a single LTR in TYR Ex1, which could be explained 


\section{Origin of AL1.9}

178 A possible interpretation of the Ex1 PCR results is that the TYR locus of the AL animal is heterozygous; one TYR allele carries the entire walb element and the other allele contains a solitary LTR. However, it needs to be considered that PCR amplification frequently results in the generation of an artifact product when the template DNA contains a repeat structure. In the case of template DNA with LTRs, a strand synthesized without sufficient elongation can be annealed to the other LTR in the next cycle and serve as a primer. Once a short fragment is generated, it is expected to have a higher amplification efficiency because of its shorter length. To evaluate this possibility, we performed a PCR assay with template DNA that mimicked diploid genomic DNAs of TYR homozygotes or TYR heterozygote (Fig. S2). We demonstrated that (i) an AL1.9 fragment is produced from the AL8.6 clone as a PCR artifact, (ii) amplification efficiency in AL1.9 is higher than that in AL8.6 to the extent that AL8.6 is not detectable in the heterozygote mimicry (Hum+LS), and (iii) the quantitative ratio of the two fragments in the product from the homozygote mimicry (Hum+LL) was similar to that from the AL genomic DNA (AL lane in Fig. 3). These results indicate that the AL animal is homozygous for the allele that corresponds to AL8.6.

\section{Search of other marsupial genomes for walb}

A BLAST search against the tammar wallaby genome database (Meug_1.0), using the entire walb_red01 sequence (7137 bp) as a query, returned a highscore hit to one sequence, and the entire walb LTR sequence (389 bp) was aligned to a 388-bp sequence with one gap and a nucleotide identity of $95 \%$ (371/388). The sequence alignment is shown later, together with the results of structural analysis. The lack of a hit to the walb internal region could be interpreted as the presence of a solitary LTR in tammar wallaby, but this sequence may be an artifact generated by a sequencing or assembly error during the database construction. We also conducted the same searches against the latest database versions of koala, wombat, Tasmanian devil, and opossum; however, a nearly complete similarity like that observed with tammar wallaby was not found. 


\section{PCR to detect LTR-encompassed regions}

212 To determine whether the internal region of walb is present in the tammar

213 wallaby genome, and to find other walb copies in the red-necked wallaby, we

214 conducted a PCR assay by devising primers suitable for this purpose. A PCR

215 assay to amplify a sequence that includes two LTRs causes an artifact product,

216 as revealed with AL1.9. To avoid this, we prepared a forward primer that

217 represented a nucleotide block in the $3^{\prime}$ half of the LTR, and a reverse primer in

218 the 5' half of the LTR (Fig. 4A). A PCR assay using these primers yielded a

219 single fragment of approximately $2.6 \mathrm{~kb}$ from genomic DNA of the red-necked

220 wallaby, and several fragments of 5 to $9 \mathrm{~kb}$ from the tammar wallaby (Fig. 4B).

221 These results suggested that the red-necked wallaby contains a walb copy of

222 approximately $2.6 \mathrm{~kb}$, and that the tammar wallaby possesses walb copies of

223 various lengths. We combined the PCR products with the plasmid vector,

224 introduced the DNA into bacteria, and picked up individual bacterial colonies.

225 Seven of the eight red-necked wallaby clones we examined were found to carry an insert fragment and all of these insert fragments were $2.6 \mathrm{~kb}$ in length. One of these was named walb_red02 and sequenced for its structure (registered in

228 GenBank with accession number LC647198). Twelve of the 16 tammar wallaby 229 clones we checked were found to have insert fragments of different lengths. Of

230 these 12 clones, four carried insert fragments of $>7 \mathrm{~kb}$ (Fig. 4C). In the

231 electrophoresis image shown in Fig. 4C, the four clones were arranged in order

232 of length and designated as walb_tam01 to walb_tam04. We were interested in

233 finding walb copies which were longer than walb_red01 (7137 bp) because

234 longer copies might have a structure closer to that of the original retrovirus. We

235 sequenced the entire insert portions of the two longest clones (walb_tam01 and

236 _tam02; registered in GenBank with accession numbers LC647196 and

237 LC647197, respectively).

\section{Structure of the internal region of the walb copies}

240 The three walb copies of $>7 \mathrm{~kb}$ in length (walb_red01, _tam01, and_tam02)

241 consisted of the same components (Fig. 5). Pairwise comparisons revealed that

242 many of the components of walb_red01 and wal_tam02 were slightly shorter

243 than those of walb tam01, and that these shorter lengths result from the lack of

244 small nucleotide blocks. This structural relationship suggested that walb_tam01

245 is a copy identical or close to a canonical endogenous retrovirus and the other

246 three copies are variant sequences derived through partial deletions. The 
247 walb_red02 was a copy in which an internal region as large as $5.5 \mathrm{~kb}$ was 248 deleted.

Three ORFs were identified in walb_tam01. Their deduced amino acid sequences were aligned, without large deletions or insertions, to those of the genes contained in TvERV (Fig. 6). The first ORF corresponded to TvERV gag, the second ORF to pro and pol, and the third ORF to env. Based on these clear correspondences, we denote the three ORFs as gag, pro/pol, and env in Fig. 5 and Fig. 6. Similar to TvERV and many other LTR retroelements, the head region of pro was overlapped with the tail region of gag, and there was a frameshift between them. A typical Lys-tRNA primer binding site was identified upstream of $g a g$, and a typical polypurine tract was found downstream of env.

The source virus of TvERV is considered to belong to the Betaretrovirus genus (Escalera-Zamudio et al. 2015). Assuming that this is also the case with walb, we constructed a phylogenetic tree of several known betaretroviruses (Fig. 7) plus walb. A gammaretrovirus (KoRV) was also added for comparison. TvERV and walb formed a cluster in this tree.

\section{Structure of LTR}

265

The walb_red01 clone contained the entire LTR sequence because the primers used for PCR amplification were those located outside the element. In contrast, in the other three clones, the $5^{\prime}$ terminal region of the $5^{\prime}$ LTR and the $3^{\prime}$ terminal region of the 3' LTR remained unknown because the PCR primers used were located inside the LTRs. To overcome this and draw available information, we analyzed the structure of the 388-bp sequence that was aligned to the walb_red01 LTR (389 bp) and identified in the tammar wallaby genome database. The CAAT box, TATA box, poly(A) addition signal, and terminal inverted repeats are standard components of retrovirus LTRs. These were all found in the two LTR sequences examined (Fig. 8).

\section{Discussion}

\section{Cause of the albino phenotype}

280 In AL8.6, the first 510-bp sequence of Ex1 was followed by the walb_red01

281 sequence. It is an acceptable assumption that during transcription of this walb-

282 carrying TYR gene, RNA polymerase moves along the template strand and 
enters the walb portion. A peptide produced by translation of mRNA generated in this manner is expected to comprise 226 amino acids because a termination codon (TAA) occurs at the 227 th site. In this peptide, the sequence of the first 170 amino acids of tyrosinase is represented, but an unrelated sequence comprising 56 amino acids follows it. Human tyrosinase consists of 530 amino acids. If we assume that the wallaby tyrosinase is similar in size, a peptide that lacks approximately the last two-thirds portion is produced from the walbcarrying TYR gene of the AL animal. This is highly probable because a typical splicing donor site (MAGGTRAGT, using the IUPAC nucleotide codes) (Zhang et al. 2003), or even its core nucleotide block (AGGT), was not found before the stop codon.

The walb_red01 LTR contains promoter signals (Fig. 8), and a transcript may be produced through the function of these signals. A peptide produced from this transcript lacks approximately the first one-third portion of the wallaby tyrosinase.

There are many reports of human albinism due to mutations in TYR (the P14679 file of the UniProtKB database). These reports include examples of oculocutaneous albinism caused by mutational amino acid changes at sites after the 170th site, including V177F, P205T, and C289G. There are also examples of albinism due to changes located before the 170th site, including H19Q, P81L, and G109R. These examples suggest that the walb insertion in TYR is the cause of the albino phenotype of the AL animal. In addition to the insertion, the AL TYR may carry other mutation(s) that affect tyrosinase function. However, the walb insertion is considered to be so critical that the insertion alone can cause a tyrosinase malfunction.

\section{Origin of the mutant TYR gene}

The AL animal (Lily) was homozygous for the walb-carrying allele. Her mother (Ran) exhibited the wild-type body color, suggesting that Ran was heterozygous and the walb-carrying allele is recessive to the wild-type allele. Because Ran is already dead and no tissue sample is available, we cannot confirm this directly. Lily's father has not been identified but is likely to have the wild-type body color. Both parents were born in a kangaroo farm in the Netherlands, which suggests that Lily's inbreeding coefficient may be high. The walb-carrying allele is thought to have already been present in a founder animal of the farm. We do not have information to estimate how long the mutant allele was maintained 
until reaching this particular founder animal; however, based on the reasoning described below, the time span is considered to be short on the evolutionary scale.

322

\section{Properties as an endogenous retrovirus}

324 The walb_tam01 copy was the longest of the four walb copies that we cloned

325

326

327

328

329

330

331

332

333

334

335

336

337

338

339

340 and analyzed. It contained three ORFs that corresponded to gag, pol/pro, and env of retroviruses, and other signaling motifs commonly found in retroviruses. Although the entire LTR regions of walb_tam01 could not be obtained because of the limitation in probe design, one can expect that walb_tam01 carries LTRs similar in sequence to those of walb_red01. Our survey of the walb_red01 LTRs revealed various motifs that are usually found in retroviruses. These structural features led to our conclusion that walb is an endogenous retrovirus, which was initially only an assumption in our study. Our phylogenetic analysis suggested a close relationship between the retrovirus from which walb originated and betaretroviruses.

\section{Cause of structural variation}

The walb_red01,_red02, and _tam02 copies were shorter in length than walb_tam01, mainly because of internal deletions. It is known that errors in template switching during reverse transcription are common and these frequently result in internal deletions (Bruner et al. 2016). The most likely cause of the emergence of the three shorter copies would be the occurrence of such errors at the time of endogenization. It is another possibility that internal deletions occurred in the DNA sequences while they were inherited as proviruses. These are not mutually exclusive; the shorter copies may have experienced multiple sequence alteration events.

\section{Origin of walb}

Our PCR experiment (Fig. 5) and subsequent cloning and sequencing analysis (Fig. 6) showed that both the red-necked wallaby and tammar wallaby harbor multiple walb copies in their genomes and these copies have sequence variations. This situation may have resulted from a walb endogenization which occurred before the divergence of the two wallaby species, that is, in their common ancestor. Another possibility is that walb was endogenized in each species after the species split. These two scenarios are not mutually exclusive; 
endogenization may have occurred both before and after the speciation.

How widely walb is distributed in the phylogeny of marsupials, or even mammals, is not known. Although our BLAST searches did not find a walb-like sequence in the available genome databases, including those of koala and opossum, this does not necessarily imply that walb is absent in their genomes. It should be noted that the BLAST search against the tammar wallaby database failed to find a walb copy, except for a single LTR sequence. Multiple walb copies do exist in the tammar wallaby genome, as demonstrated in our PCR and sequencing experiments. This discrepancy may have been caused by the tendency of repetitive sequences to be underrepresented in genome databases (Koga 2012).

\section{Recent insertion}

Considering that walb is an endogenous retrovirus, we can propose the following three hypotheses concerning the integration of walb_red01 into TYR: (i) endogenization: an individual of the red-necked wallaby was infected by a virus that was produced in another individual or in another host species, and the virus DNA was inserted into the $T Y R$ of a germline cell; (ii) reinfection: a virus produced from a previously endogenized provirus infected the same host individual and DNA was inserted into the TYR of a germline cell; and (iii) retrotransposition: a previously endogenized provirus was transcribed and reverse transcribed, and the DNA product was inserted into $T Y R$, all occurring within a single germline cell. The second hypothesis involves reinfection solely by the provirus and reinfection with participation of an exogenous virus. We do not have sufficient information to consider which of these is most likely to have occurred. In any of these cases, however, it can be inferred that this germline insertion event occurred recently on an evolutionary timescale based on two observations.

One is the linkage between walb_red01 and the mutant TYR allele, the latter causing oculocutaneous albinism. Mutant animals with oculocutaneous albinism generally have serious disadvantages in terms of survival in wild circumstances, because of a higher chance of detection by predators, higher chance of escape of prey, decrease in UV protection, and decrease in visual acuity. These disadvantages result in the exclusion of albino mutant alleles from the gene pool of the host organism. Thus, even if an albino allele emerges through mutation, it does not persist long in the population of the host organism. This implies that 
391 when an existing albino allele is found, its origin is expected to be recent. If the 392 mutant allele carries an insertion sequence, the time span from the occurrence of 393 insertion is expected to be short. Although albino mutant alleles are mostly 394 recessive to wild-type alleles, the recessive nature does not invalidate the above 395 explanation. Suppose that a recessive allele emerges, and that strong natural 396 selection acts against its homozygous carrier. If the genotype frequency of the 397 heterozygote is increased, the frequency is driven back by the selection pressure 398 to eliminate the mutant homozygote. When the genotype frequency of the 399 heterozygote is low, the mutant allele is constantly exposed to a chance of 400 stochastic loss.

401 Another observation that supports the inference of recent occurrence is that 402 the LTRs of walb_red01, both $389 \mathrm{bp}$ in length, were identical in sequence. In 403 relation to this, analysis of LTRs of additional walb copies may provide useful 404 information on whether the insertion of walb_red01 into TYR occurred uniquely 405 in the genome or it was one of multiple events that occurred simultaneously or 406 as a series of events.

407

408

409 Author contributions

410 A.K. conceived of the study and designed the experiments. K.S. and Y.H. 411 conducted animal care and sample collection. A.K. and S.H. carried out the 412 experimental work. S.H. and Y.K. conducted data analysis.

\section{Competing interests}

415 The authors declare that they have no competing interests.

\section{Acknowledgements}

418 We are grateful to Kuragaike Park Zoo (Toyota City, Japan) for providing fecal 419 samples of the tammar wallaby. We also than Yuki Enomoto (Kyoto University) 420 for technical assistance. This work was supported by Grants-in-Aid from the 421 Japan Society for the Promotion of Science (grant number 19H03311 to A.K.).

\section{Supplementary material}

424 This article contains electronic supplementary material (Table S1, Fig. S1, Fig. 425 S2). 


\section{References}

Baillie, G.J., and Wilkins, R.J. 2001. Endogenous type D retrovirus in a marsupial, the common brushtail possum (Trichosurus vulpecula). J. Virol. 75, 2499-2507. (doi: 10.1128/JVI.75.5.2499-2507.2001)

Bannert, N, and Kurth, R. 2006. The evolutionary dynamics of human endogenous retroviral families. Annu. Rev. Genomics Hum. Genet. 7, 149173. (doi: 10.1146/annurev.genom.7.080505.115700)

Boeke, J.D., and Stoye, J.P. 1997. Retrotransposons, Endogenous Retroviruses, and the Evolution of Retroelements. In: Coffin, J.M., Hughes, S.H., and Varmus, H.E., editors. Retroviruses. Cold Spring Harbor (NY): Cold Spring Harbor Laboratory Press

Bruner, K.M., Murray, A.J., Pollack, R.A. et al. 2016. Defective proviruses rapidly accumulate during acute HIV-1 infection. Nat. Med. 22, 1043-1049. (doi: 10.1038/nm.4156)

Costas, J., 2001. Evolutionary dynamics of the human endogenous retrovirus family HERV-K inferred from full-length proviral genomes. J Mol Evol. 2001 Sep;53(3):237-43. doi: 10.1007/s002390010213. PMID: 11523010.

Eickbush, T.H., and Jamburuthugoda, V.K. 2007. The diversity of retrotransposons and the properties of their reverse transcriptases. Virus Res. 134, 221-234. (doi:10.1016/j.virusres.2007.12.010)

Koga, A. 2012. Under-representation of repetitive sequences in whole-genome shotgun sequence databases: an illustration using a recently acquired transposable element. Genome 55, 172-175. (doi: 10.1139/g11-088)

Körner, A., and Pawelek, J. 1982. Mammalian tyrosinase catalyzes three reactions in the biosynthesis of melanin. Science 217 (4565), 1163-1165.

Kumar, S, Stecher, G., Li, M., Knyaz, C., and Tamura, K. 2018. MEGA X: Molecular Evolutionary Genetics Analysis across computing platforms. Mol. Biol. Evol. 35, 1547-1549. (doi: 10.1093/molbev/msy096)

Lander, E.S., Linton, L.M., Birren, B. et al. (International Human Genome Sequencing Consortium). 2001. Initial sequencing and analysis of the human genome. Nature 409, 860-921. (doi: 10.1038/35057062)

Llorens, C., Muñoz-Pomer, A., Bernad, L. et al. 2009. Network dynamics of eukaryotic LTR retroelements beyond phylogenetic trees. Biol. Direct 4, 41. (doi.org/10.1186/1745-6150-4-41)

Paces, J., Pavlícek, A., Zika, R., Kapitonov, V.V., Jurka, J., and Paces, V. 2004. 
462 HERVd: the Human Endogenous RetroViruses Database: update. Nucleic 463 Acids Res. 32, D50. (doi: 10.1093/nar/gkh075)

464 Vargiu, L., Rodriguez-Tomé, P., Sperber, G.O., et al. 2016. Classification and 465 characterization of human endogenous retroviruses; mosaic forms are 466 common. Retrovirology 22, 7. (doi: 10.1186/s12977-015-0232-y) 467 Vitte, C., and Panaud, O. 2003. Formation of Solo-LTRs through unequal 468 homologous recombination counterbalances amplifications of LTR 469 retrotransposons in rice Oryza sativa L. Mol. Biol. Evol. 20, 528-540. (doi: $470 \quad 10.1093 / \mathrm{molbev} / \mathrm{msg} 055)$

471 Zhang, X.H., Heller, K.A., Hefter, I., Leslie, C.S., and Chasin, L.A. 2003. 472 Sequence information for the splicing of human pre-mRNA identified by 473 support vector machine classification. Genome Res. 13, 2637-2650. 474 (https://doi.org/10.1101/gr.1679003) 
475

476

477

478

479

480

481

482

483

484

485

486

487

488

489

490

491

492

493

494

495

496

497

498

499

500

501

502

503

504

505

506

507

508

509

510

\section{Figure legends}

Fig. 1. Wallaby animals. The wild-type (WT) animal has black eyes and a body color of the regular pigmentation pattern of the red-necked wallaby. The albino (AL) animal exhibits oculocutaneous albinism, having red eyes, white hair, and pinkish skin. Their body height is approximately $80 \mathrm{~cm}$.

Fig. 2. Results of PCR amplification for $T Y R$ exon regions. The structure of human TYR is shown at the top. Green squares are the exons (Ex1 to Ex5) and the grey bar indicates introns or untranscribed regions. Orange triangles indicate the location and direction of PCR primers to amplify each exon region. The elements do not strictly reflect their sizes or positions. Electrophoresis photographs of PCR reaction mixtures are shown below. The lanes are: $\mathrm{M}$, size marker; WT, WT template; AL, AL template, and N, no template DNA. The expected product size based on the tammar wallaby or koala sequence information is shown below each photograph.

Fig. 3. Structure of Ex1 PCR products. The photograph of Ex1 PCR products is shown here again. Each product fragment was cloned and sequenced, and analyzed for structure. The size in bp of each component is shown in parentheses.

Fig. 4. PCR to amplify LTR-encompassed regions. A. Locations and directions of the PCR primers. The yellow triangles indicate the LTRs. PrF and PrR represent nucleotide blocks in the LTR sequence as shown here. The exact locations of $\operatorname{PrF}$ and $\operatorname{PrR}$ were nt 202 to 236 and nt 150 to 116, respectively, on the walb sequence (GenBank LC631488) in which the 5' LTR consisted of nt 1 to 389. B. PCR from genomic DNA. The template DNA was genomic DNA of the two wallaby species. C. PCR from walb clones. The template DNA was the walb clone that originate from the red-necked wallaby, and the four clones from the tammar wallaby that exhibited fragment lengths of $>7 \mathrm{~kb}$.

Fig. 5. Structure of the walb clones. The walb_red01 copy was cloned by PCR using primers that represented TYR (see Fig. 2). Because these primers were located outside of the walb element, the clone obtained included the two entire LTRs. The PCR primers for the other three clones were located inside the LTRs 
511 (see Fig. 4) and therefore, the walb terminal regions were not included in the 512 clones obtained. The longest clone was walb_tam01, and the other three clones 513 lacked one or more portions when compared with walb_tam01. The deleted 514 regions are shown by dotted boxes. The blue triangle on walb_red01 indicates 515 an extra nucleotide in comparison to walb_tam01. The ORFs represented by red 516 arrows are those identified in the sequence of each clone. Because the ranges of 517 ORFs are affected by deletions or other sequence variation, the identity of the 518 ORFs among the clones was not taken into consideration. The sequences of 519 regions $\mathrm{a}$ and $\mathrm{b}$ regions are shown at the bottom, with sequence motifs present 520 therein.

522 Fig. 6. Dot matrix analysis of amino acid sequences. Reduced amino acid 523 sequences from the walb_tam01 ORF nucleotide sequences (horizontal axis) 524 were compared with those of gag, pro, pol and env of TvERV (vertical axis).

525 The criteria were a match of three or more amino acids over a window of 10 526 amino acids. The axes are marked with an interval of 100 amino acids.

528 Fig. 7. Phylogenetic tree of betaretroviruses, a gammavirus, and walb. Using the 529 MEGA X platform (Kumar et al. 2018), amino acid sequences corresponding to 530 the pol gene were aligned by the MUSCLE program and their phylogenetic tree 531 was constructed by the maximum likelihood method. The bootstrap values were 532 obtained through 1000 repetitions. The table under the tree shows the host 533 organism of the viruses analyzed and their GenBank accession numbers. KoRV 534 is a gammavirus, which was included in the analysis for comparison.

536 Fig. 8. Alignment of LTR sequences and motifs found therein. The upper line 537 (labelled red LTR) is the sequence of the LTR that was contained in the 538 walb_red01 clone. The lower line (tam LTR) is the sequence identified by a 539 BLAST search of the tammar wallaby genome database. The dot indicates the 540 occupation of the site by an identical nucleotide. The minus symbol implies 541 absence of a site at its position. The motifs found are shown by purple boxes.

542 The underline indicates 6-bp blocks that are considered to be target site 543 duplications. 

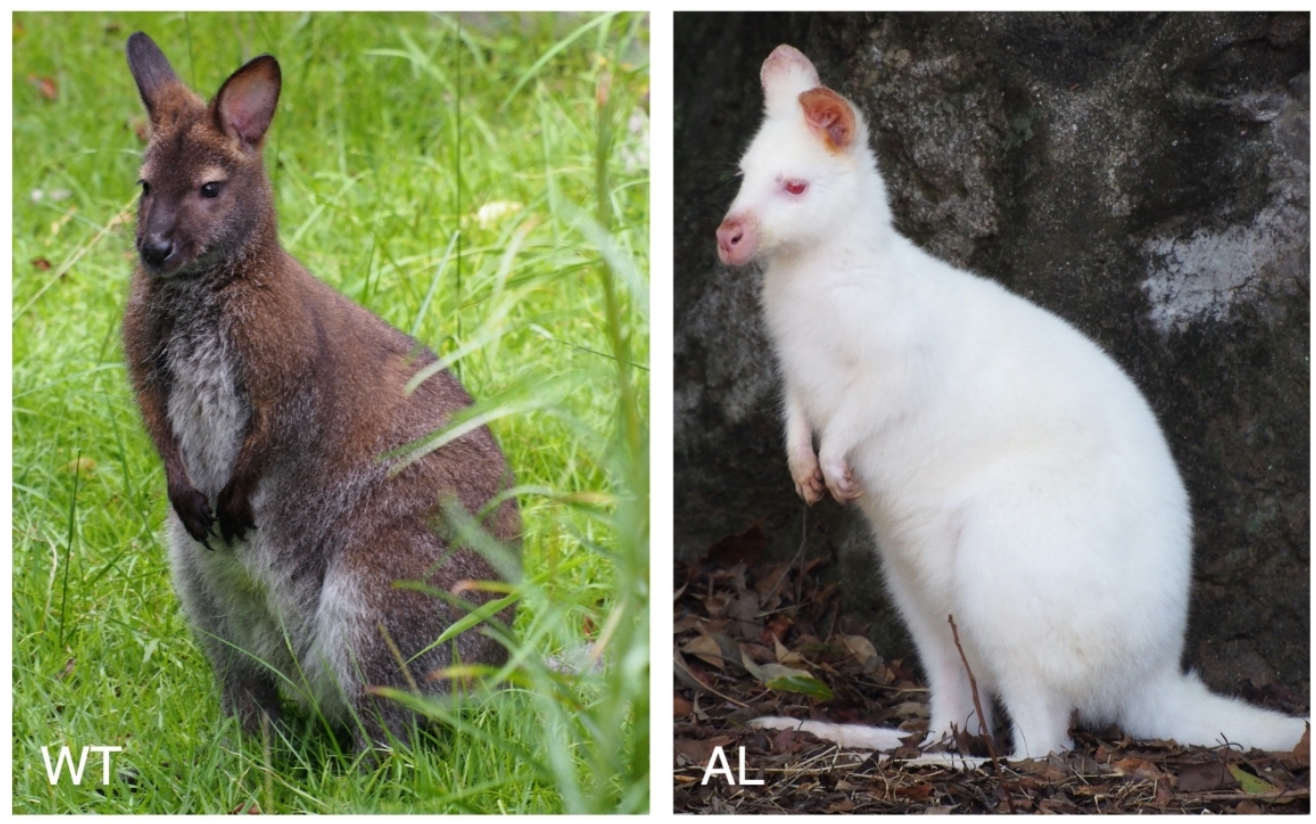

Fig. 1

149x93mm (358 x 358 DPI) 


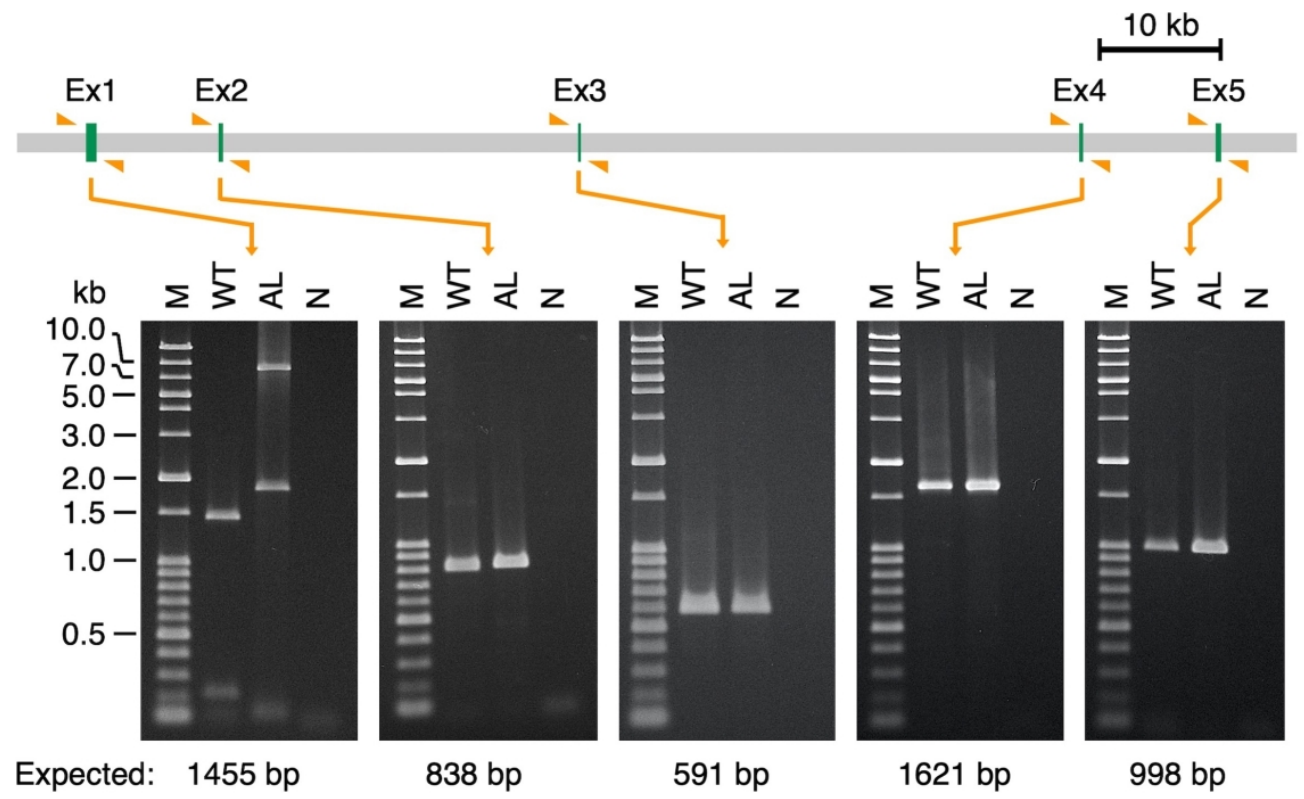

Fig. 2

$149 \times 91 \mathrm{~mm}(399 \times 399 \mathrm{DPI})$ 


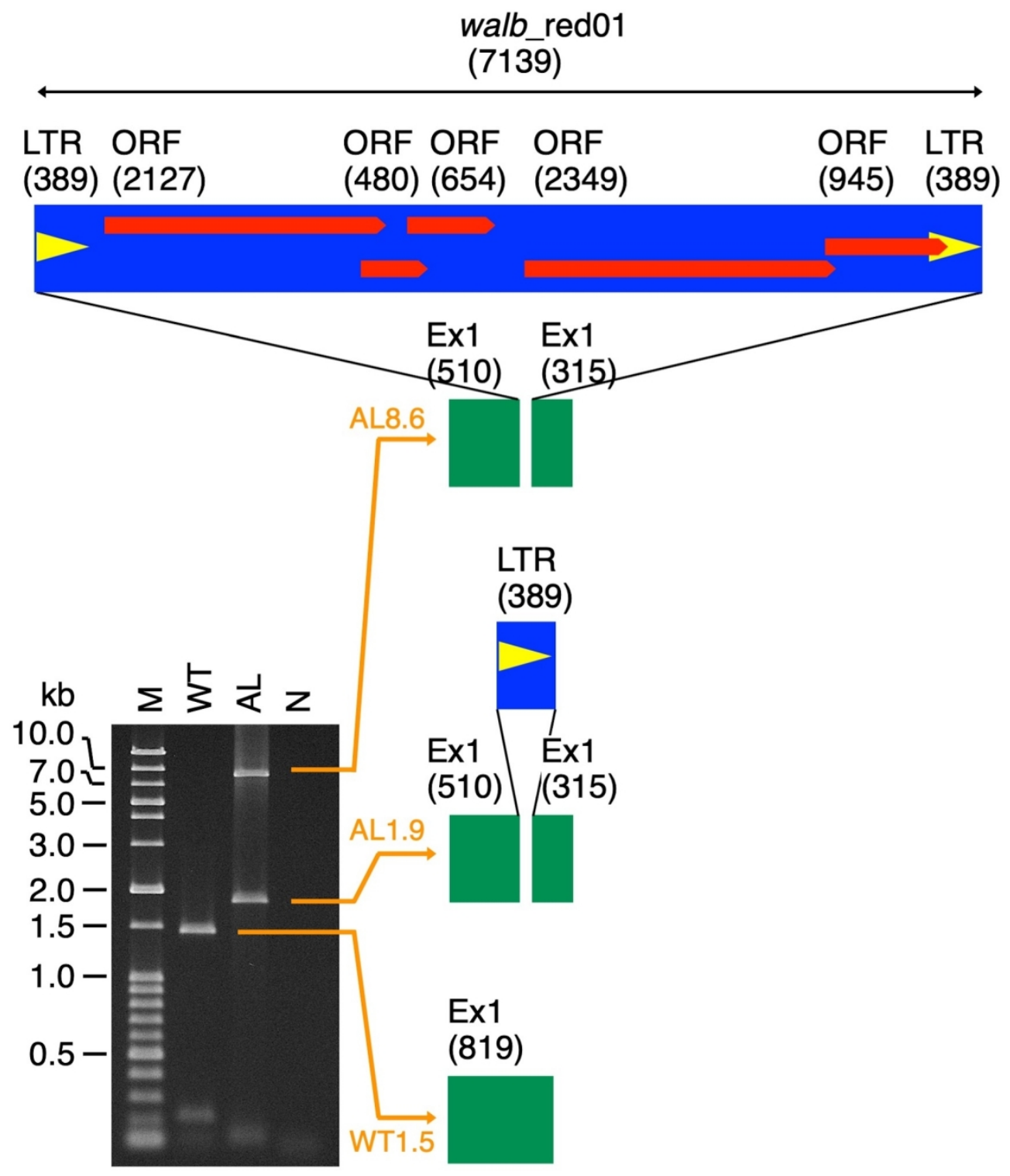

Fig. 3

$149 \times 174 \mathrm{~mm}(295 \times 295 \mathrm{DPI})$ 


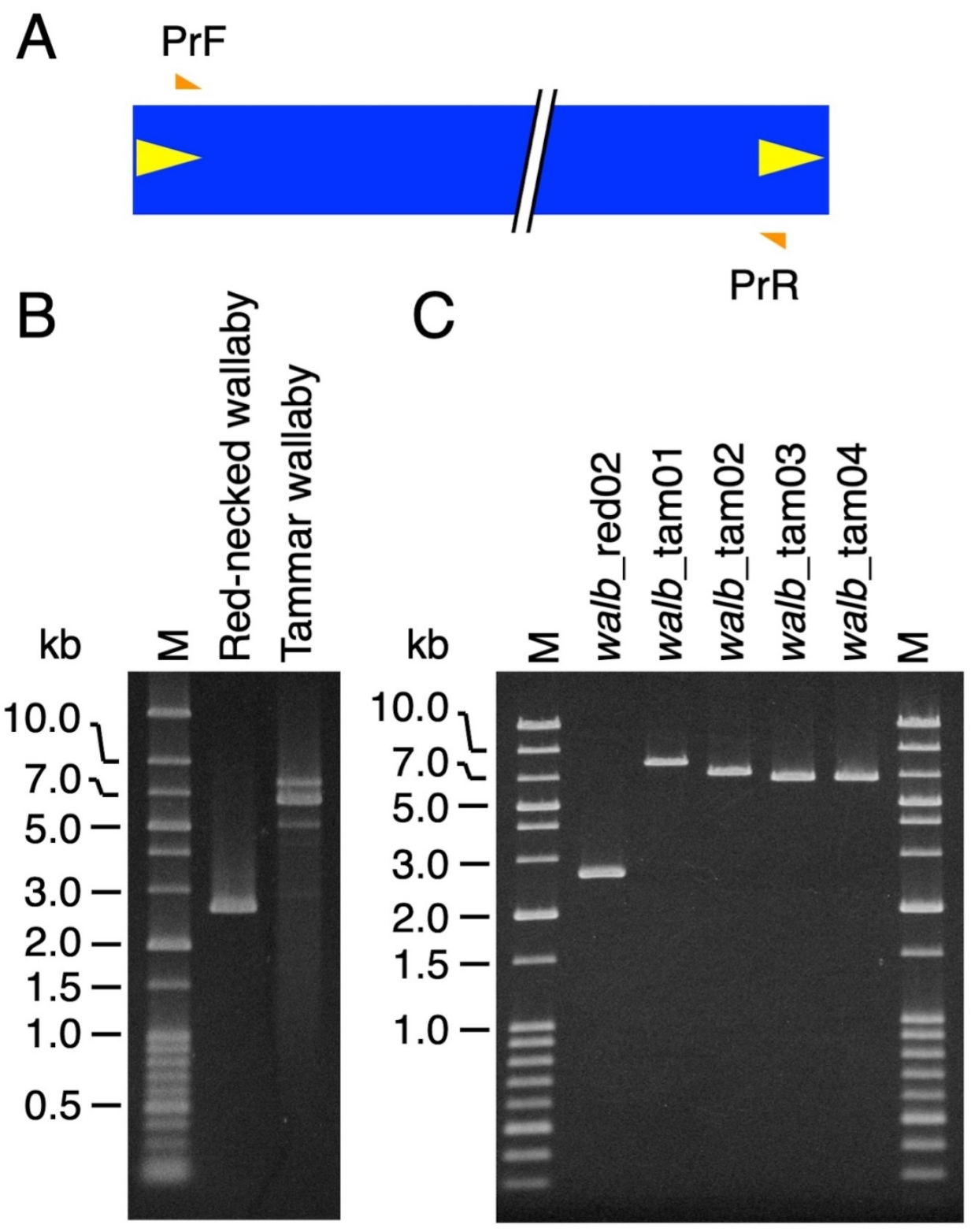

Fig. 4

$129 \times 162 \mathrm{~mm}(265 \times 265 \mathrm{DPI})$ 


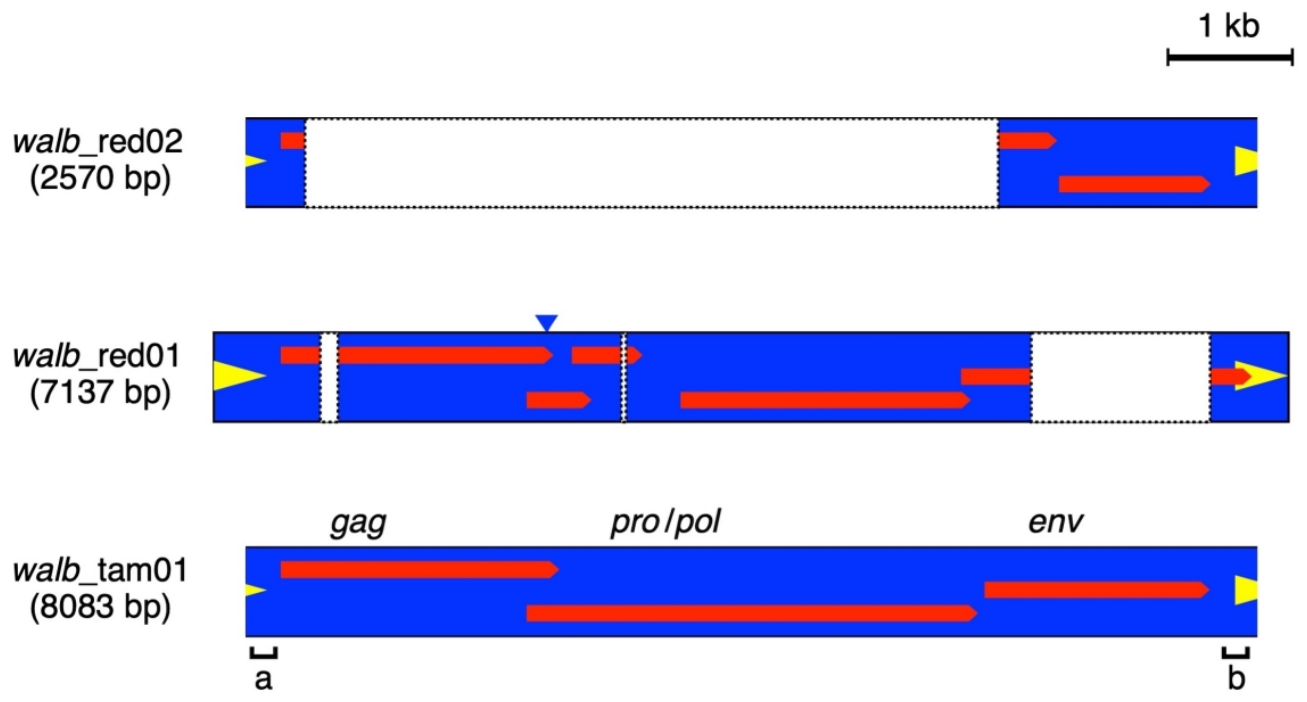

walb_tam02

(7081 bp)
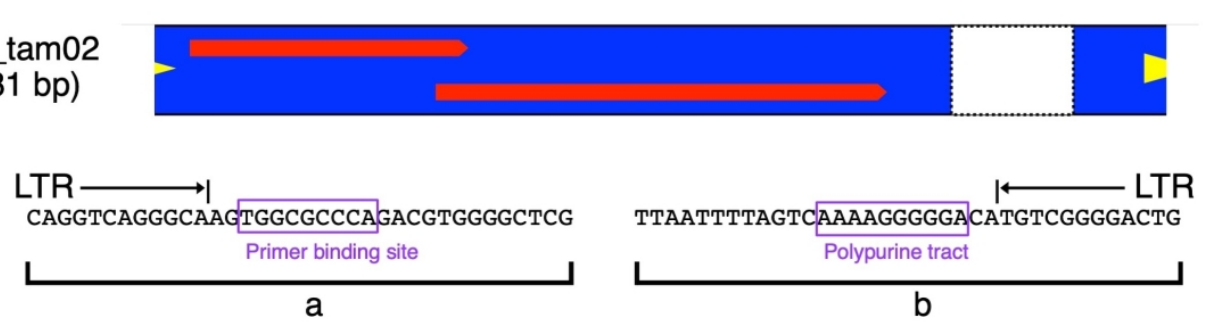

Fig. 5

$149 \times 123 \mathrm{~mm}(376 \times 376$ DPI) 

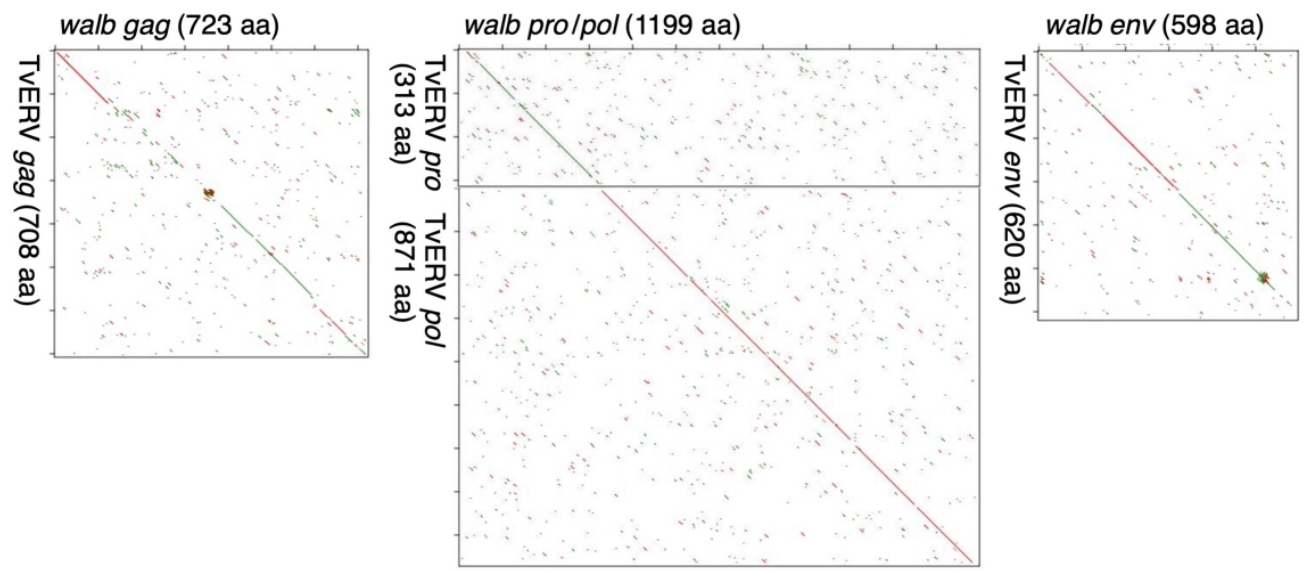

Fig. 6

$149 \times 65 \mathrm{~mm}(454 \times 454$ DPI $)$ 

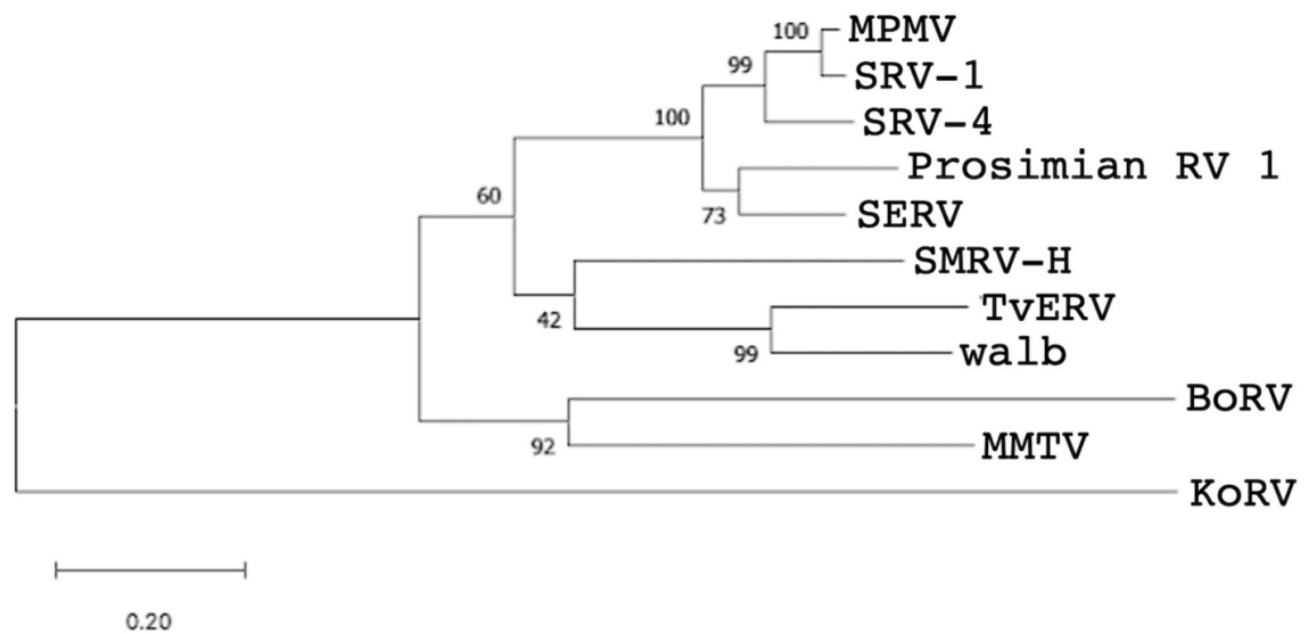

\begin{tabular}{|c|c|c|}
\hline Virus & Host organism & GenBank \\
\hline TVERV & brushtail & AF224725 \\
\hline MMTV & mouse & AF 228552 \\
\hline MPMV & rhesus macaque & $\mathrm{AF} 033815$ \\
\hline SERV & baboon & U85505 \\
\hline SRV-1 & rhesus macaque & M11841 \\
\hline SMRV-H & human & M23385 \\
\hline BORV & bovine & NC_029852 \\
\hline Prosimian RV 1 & lemur & MT787217 \\
\hline SRV-4 & crab-eating macaque & NC_014474 \\
\hline KoRV & koala & NC_039228 \\
\hline walb & wallaby & LC6 47196 \\
\hline
\end{tabular}

Fig. 7

119x119mm ( $300 \times 300$ DPI) 


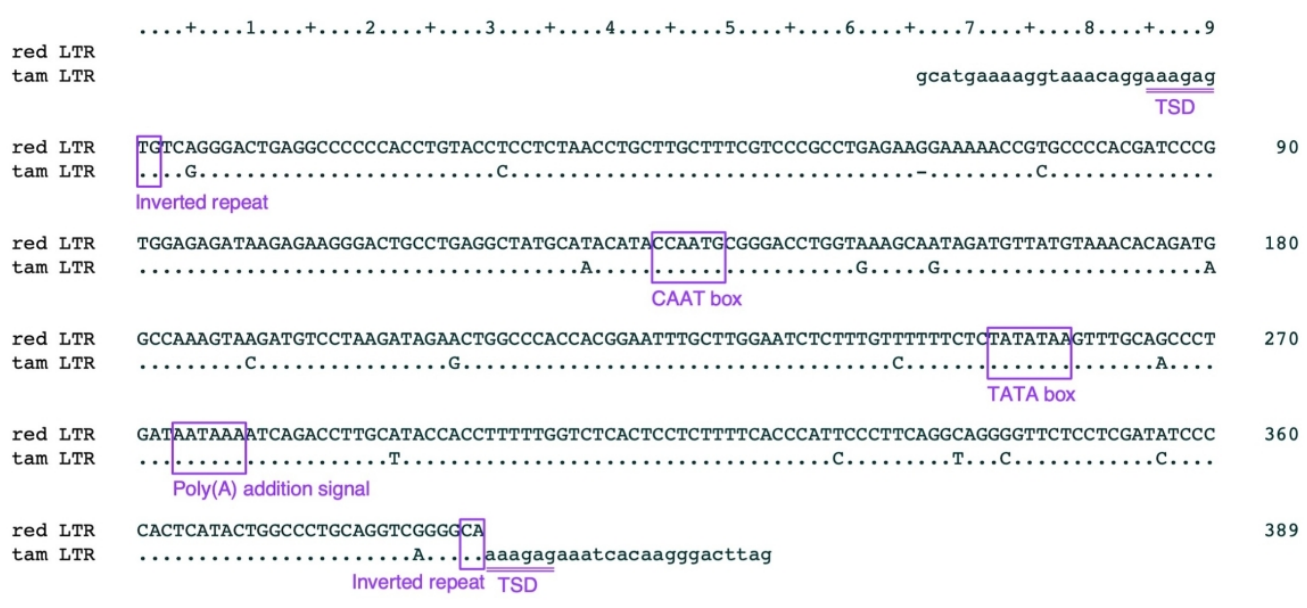

Fig. 8

$150 \times 68 \mathrm{~mm}(394 \times 394 \mathrm{DPI})$ 